Finisterra, XXXVI, 72, 2001, pp. 127-139

\title{
L'ART DU PAYSAGE ET LE GÉOGRAPHE
}

A. $\operatorname{HuFtY}^{1}$

\begin{abstract}
Résumé - Les artistes sont des acteurs géographiques quand ils transforment une toile en territoire; pendant plus de cinq siècles en occident, ils y ont également matérialisé leur perception du monde visible et ainsi inventé le paysage. La fréquentation des arts est alors une source de connaissance pour les géographes pour comprendre le versant sensible de notre rapport à l'espace visible et ses variations historiques.

Mots-clés: pensée géographique, cartographie, épistémologie, perception, aménagement du territoire, paysage.

Summary-ART OF LANDSCAPE AND GEOGRAPHY - An artist is a geographic actor who transforms a canvas into a territory, which reflects both materially and symbolically the spatial ideas of an epoch. The planning process is influenced by the style of earlier works of art. Contact with art is not a luxury for geographers.

Key words: Geographic thought, cartography, epistemology, perception, planning, landscape.

Resumo - A ARTE DA PAISAGEM E O GEÓGRAFO - Um artista é um actor geográfico, quando transforma uma tela num território que reflecte as concepções espaciais de uma época, tanto do ponto de vista material, como do ponto de vista simbólico. O processo de planeamento é influenciado pelo estilo de anteriores obras de arte e o contacto com a arte não é um luxo para os geógrafos.

Palavras-chave: pensamento geográfico; cartografia; epistemologia; percepção; planeamento; paisagem.
\end{abstract}

\section{INTRODUCTION}

Le milieu où vit toute société humaine a toujours été observé, perçu, intériorisé, mais rarement représenté par une image, construite sur l'illusion de la perspective, image nommée, appelée depuis cinq siècles environ dans l'art occidental, un paysage.

Beaucoup de lieux, le plus souvent associés à des événements, avaient été décrits et illustrés depuis l'antiquité sans que pour autant on parle de paysage; il a fallu attendre le $16^{\mathrm{e}}$ siècle pour que naisse une "sensibilité proprement

1 Département de Géographie, Université Laval, Québec. E-mail : andre.hufty@ggr.ulaval.ca 
paysagère» (BERQue,1995). À l'ivresse géographique qui accompagnait les grandes explorations de la Terre jusqu'au $18^{\mathrm{e}}$ siècle a correspondu le paysage pictural composé (LHOTE, 1948), qui montrait des organisations du monde en rassemblant des fragments imaginaires ou rencontrés au cours des voyages. Puis progressivement les vues partielles vont l'emporter. Un besoin de «sensations nouvelles expérimentées au contact de la Nature amène à penser et à produire la peinture de paysage comme une source d'effets surprenants sur le spectateur» (Lнот, 2000); le pittoresque et le sublime multiplient les vues partielles et favorisent le pleinarisme; pendant les $18^{\mathrm{e}}$ et $19^{\mathrm{e}}$ siècles naissent de nombreuses formes nouvelles : c'est la découverte de la montagne, des rivages marins, des pays exotiques et du nouveau monde; la campagne humanisée, un moment délaissée, va connaître un regain d'attention avec les fiertés nationalistes, quelque peu nostalgique d'ailleurs autour des grandes villes,

$\mathrm{Au} 20^{\mathrm{e}}$ siècle, même si les acteurs et les moyens de représentation ont changé, nous restons sensibles à l'apparition de nouveaux paysages. Donnons comme exemple l'invention récente du marais par les écologistes, dont la présentation filmée avec ses vues successives recrée momentanément des conditions propices à une méditation en face d'un coin de pays; et pourquoi pas la photographie nocturne d'un coin de Terre vu par satellite, semblable à une constellation d'étoiles? Cependant les réussites sont rares. La création ou la reconnaissance d'un paysage est difficile car plusieurs conditions doivent être réunies. Contempler son coin de terre ne suffit pas à faire naître une sensibilité paysagère; elle a besoin d'un médiateur qui rende visible la relation entre moi et le milieu. L'art joue encore ce rôle mais d'une manière détournée comme dans le «land-art» où le cadre pictural a disparu au profit d'une trace, d'une sorte de sculpture qui défigure la Nature et qui préfère l'action à la contemplation (GARRAUD,1994). Le support matériel n'est d'ailleurs peut-être plus nécessaire pour appréhender une portion de territoire comme un paysage; le support est intériorisé grâce à des siècles d'éducation qui ont accumulé des cadres d'images mentales.

Cette lecture devrait faire partie du bagage du géographe. Il a tendance à baptiser "paysage» le moindre coin de terre qui lui sert à illustrer une théorie économique ou fonctionnelle. Cette voie confond l'environnement, appellation cartésienne de notre relation fonctionnelle avec le milieu, avec le paysage qui en est le versant sensible. D'autres regards l'aident à découvrir de nouveaux chemins. La peinture de paysage est une de ces sources de connaissances, qui éduquent le regard et l'esprit (Piveteau, 1989). Nous allons donc explorer ce terrain en survolant quelques œuvres.

\section{ANALYSE DE QUELQUES CEUVRES}

Le réservoir d'images symboliques du moyen âge qui racontent des histoires à propos de la maison de Dieu est progressivement envahi par le monde exté- 


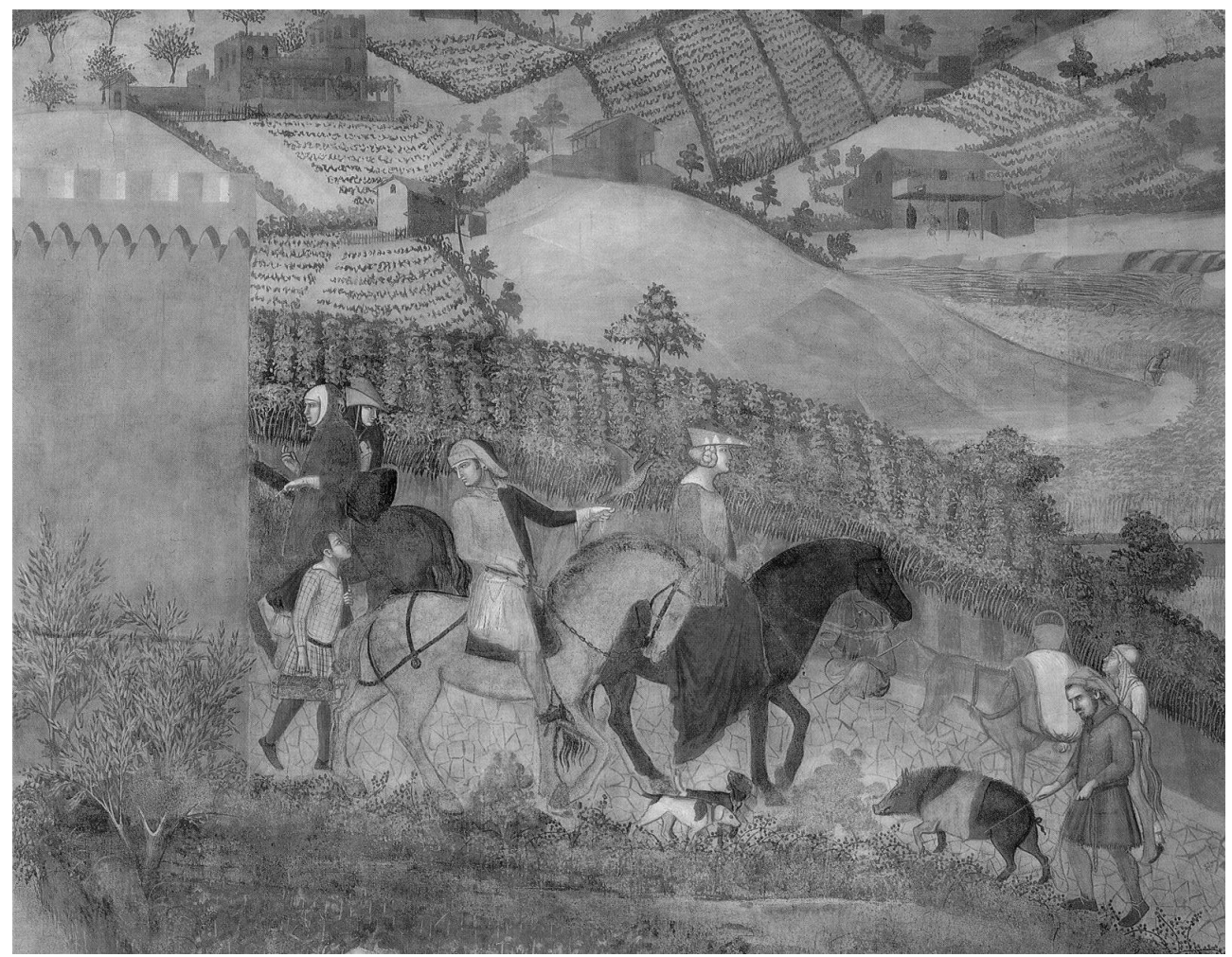

Fig. 1 - Les échanges ville-campagne, extrait du «Bon gouvernement», Ambregio Lorenzetti 1337-1339, fresques, palais communal de Sienne.

Fig. 1 - As trocas cidade-campo. Ambregio Lorenzetti 1337-1339, frescos, Siena.

rieur aux $13^{\mathrm{e}}$ et $14^{\mathrm{e}}$ siècles. Le "Bon gouvernement» de Lorenzetti (RANDOLPH, 1994), peint en Toscane vers 1330, montre que la volonté de l'homme l'emporte désormais sur l'humilité en face de la providence. L'orgueil de la cité libre de Sienne éclate dans cette véritable monographie géographique. C'est une composition réaliste, qui utilise une perspective à plusieurs points de vue (BARRE et FLOCON, 1968), et mélange différentes saisons, pour nous faire comprendre ce qui fait la richesse : les multiples travaux et activités des citoyens, rendus possibles par une sage administration. En particulier, sur la figure 1, la main du fauconnier, les pointes de deux chapeaux, la tête du premier cheval, les personnes de qualité qui quittent la ville s'opposent au groin du cochon, au dos de l'âne et au voyageur. Ce flux de personnes et de marchandises qui unissent en tous sens la ville et sa campagne est un lien pictural très puissant entre les masses des murailles traitées par des lignes droites et l'étendue de la Nature où s'entrelacent les courbes, où des formes régulières s'impriment dans les 
colline, une organisation régulière des vignobles et le réseau des chemins et des haies témoignent de la bonne gestion (SERENI, 1964).

Entre le $15^{\mathrm{e}}$ et le $18^{\mathrm{e}}$ siècles, le paysage composé est à son apogée. Les tendances sont tellement nombreuses qu'il serait présomptueux d'en présenter un aperçu (CARLI, 1980; CLARK, 1988). La perspective venue d'Italie et la gradation de la luminosité venue du Nord servent à maittriser une nature humanisée, généralement vue à partir d'un point unique. Nous en donnons un seul exemple, (exposition, 1994) «Le temps calme» de Nicolas Poussin (1651, fig. 2). C'est un grand paysage construit, idéal, une interprétation de nos relations avec la Nature. À l'avant-plan, un berger laisse ses chèvres s'égailler en zig-zag : c'est un personnage médiateur, issu d'un monde intermédiaire, entre les contraintes de la civilisation et des forces qu'il faut apprivoiser. Les références au bosquet d'arbres viennent d'une lointaine tradition pastoraliste. Puis, un lac calme, dans lequel se reflètent des constructions géométriques, la défense et le labeur des hommes soulignés par un troupeau de moutons très ordonné. Et enfin la sauvagerie lointaine, un volcan et des nuages: c'est la turbulence et le jaillissement des éléments qui précèdent l'homme. Belle leçon d'aménagement du territoire! On est assez proche des paysages humanisés des rizières de P. Gourou, de l'esthétique en géographie et des «beaux» paysages ruraux à conserver.

Entre le début du $18^{\mathrm{e}}$ et la fin du $19^{\mathrm{e}}$ siècles, le développement des sciences naturelles et l'exploration du monde entier vont de pair avec une abondante production d'images dessinées ou peintes, qui se poursuivra après l'invention de la photographie. Les peintres de la nature ont abordé quantité de genres qui sont proches des préoccupations des géographes: grands paysages végétaux didactiques, les descriptions "pittoresques» des voyageurs, par exemple du grand tour d'Italie (SIMOËNS, 1994), l'observation fine de multiples points de vue par les aquarellistes ... Une vision «sublime» de la nature s'accompagne de commentaires sur la géologie, les plantes ou les nuages (CARUS, 1810). Un écologisme précoce inspire les écoles du Nord, notamment les romantiques allemands, et influence les grands paysages américains (McSHINE, 1972).

La montée du nationalisme est soutenue par des portraits de régions géographiques, mouvement parallèle aux grandes monographies régionales. Les paysages régionaux subsisteront longtemps dans un genre mineur, par exemple les vues de l'Ardenne belge ou de Charlevoix au Québec, auxquels beaucoup de gens s'identifient encore. Clarence Gagnon a contribué à fixer des images d'un Québec rural qui deviendront des archétypes dans la mémoire collective et qui seront suivies par des milliers d'épigones toujours actuels et de moins en moins originaux. La figure 3 (Village laurentien, vers 1928) est une vue fragmentaire et pittoresque d'un village paisible blotti à l'abri de ses montagnes; la perspective subsiste, soulignée de façon très conventionnelle par un traîneau mais le «schématisme des formes... les couleurs vives et contrastées...» (TréPANIER, 1997) témoignent d'influences post-impressionnistes. Cette valorisation d'une campagne humanisée coöncide avec un appel à une mise en valeur du patrimoine et au regard scientifique de l'ethnologue sur un monde 


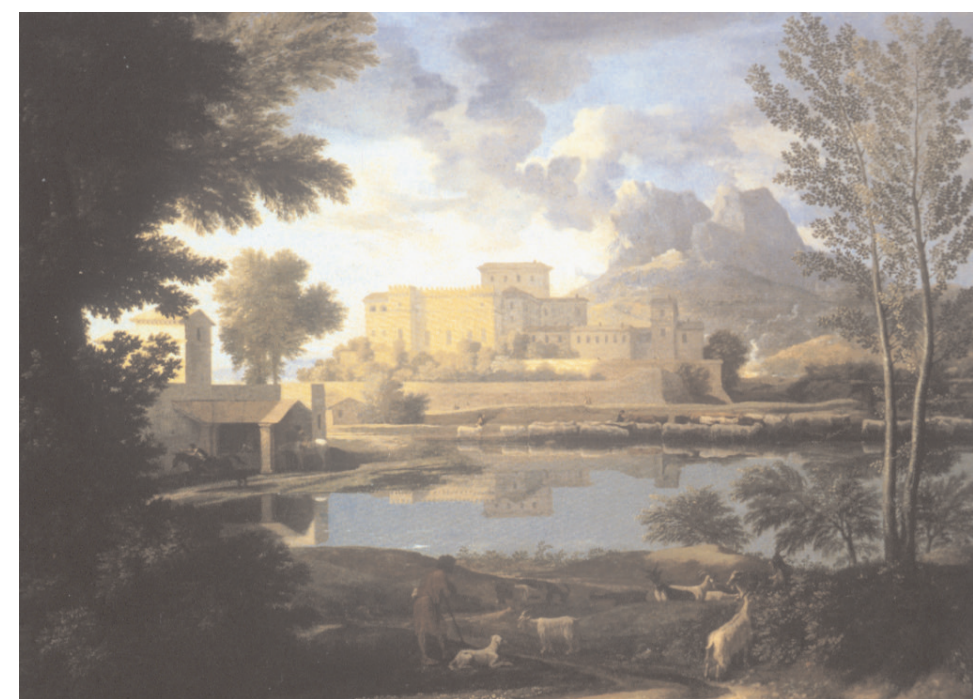

Fig. 2 - L'aménagement idéal du paysage: Le temps calme, Nicolas Poussin, 1651, huile, $97 \times 31 \mathrm{~cm}$, collection Sudeley Castle.

Fig. 2 - O ordenamento ideal do território: tempo sereno, óleo de Nicolas Possin, 1651,97×31 cm, colecção Sudeley Castle.

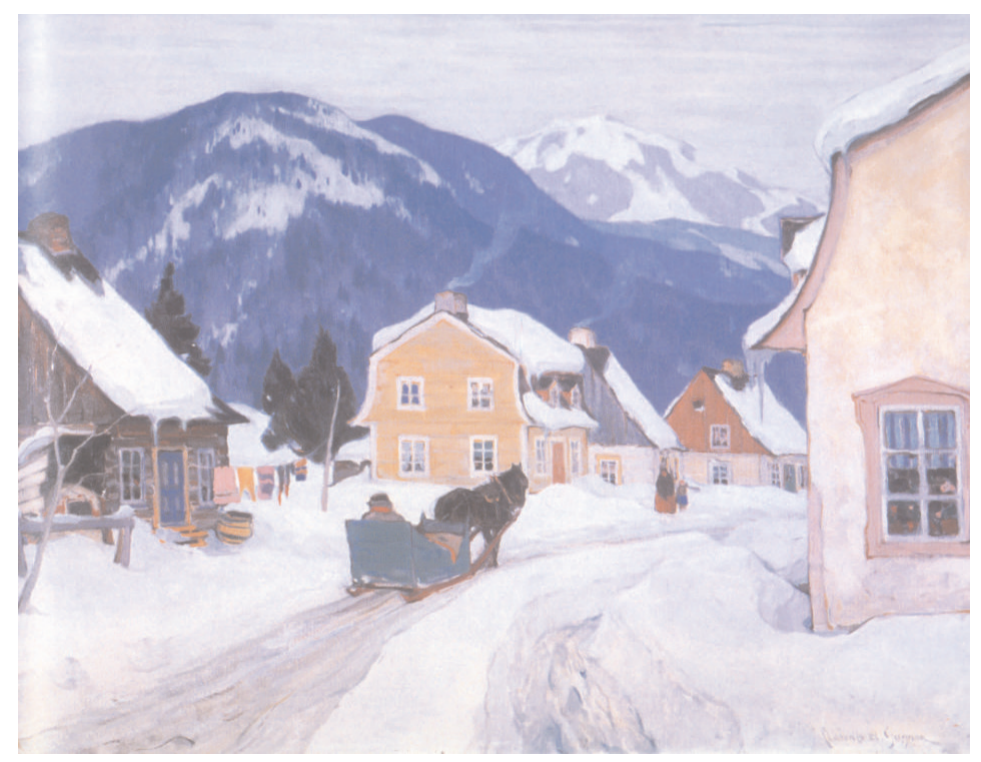

Fig. 3 - Village laurentien, Clarence Gagnon, circa 1928, huile, $73 \times 92 \mathrm{~cm}$, Musée du Québec

Fig. 3 - Aldeia da região das Laurentides (Canadá), Clarence Gagnon, cerca de 1928, óleo, 73 ×92 cm, Musée du Québec. 
paysan qui disparaît; la génération suivante des géographes étrangers va d'ailleurs s'en inspirer, avec "L'homme et l'hiver» de Deffontaines ou le «Canada français» de Blanchard. L'idée de l'aménagement esthétique de la Nature par une civilisation rurale fera partie du bagage des géographes de la première moitié du $20^{\mathrm{e}}$ siècle.

On ne peut que brièvement aborder la période actuelle qui a débuté avec la rupture de la fin du $19^{\text {e }}$ siècle. À l'image de la Nature s'est substituée l'image de nos rapports avec la Nature et une réflexion sur le pouvoir de l'image elle-même. L' œuvre artisanale fabriquée lentement a été remplacée par une explosion d'images fragmentaires, qui vont de la photographie à la simulation virtuelle animée produite par ordinateur. La peinture est devenue un discours sur le monde, qui exprime l'étonnement ou l'inquiétude personnelle en face d'un «environnement» (finis les «milieux géographiques»!), que ce dernier soit naturel, à encadrer ou à préserver peut-être parce qu'il est de plus en plus méconnu ou qu'il soit artificiel et, dans ce cas, vu comme éphémère parce que ses changements trop rapides ne laissent pas le temps de l'apprivoiser. La perception de l'expressionnisme, du surréalisme, du Pop Art ou du Land Art ne devrait pas laisser le géographe indifférent; en paraphrasant Grimaldi, on peut penser que les futurs aménagements n'auront de style qu'en reproduisant celui des œuvres actuelles.

James Turrel (fig. 4) explore ces frontières de la perception et isole un coin de ciel pour être encore capable de le contempler. Si les anciens peintres laissaient entrevoir la nature au travers d'une "veduta», nous la voyons au travers d'un écran - pensons à la télévision -, par le moyen d'une théorie ou d'un appareil. C'est la carte du géographe qui superpose des multiples grilles de lectures en espérant que de leur juxtaposition analytique naîtra une idée du monde (SAUVAGEOT, éd., 1993).

René Derouin (fig. 5) montre une autre tendance de l'art contemporain, l'abstraction des formes et des forces, de l'ordre et du chaos qui sont en résonance avec la physique actuelle. Un matériau pâteux semble injecté contre un bloc solide, faisant penser à des turbations, des déformations d'une boue littorale. Même si l'artiste (Derouin,1997) a médité sur des vues aériennes de la géologie du nord du Québec, il n'en présente pas moins une construction purement imaginaire et picturale. Des textures se répondent, une dynamique de lignes de forces les transforme peu à peu. Cette figuration est évidemment très proche de la cartographie actuelle des lignes de forces, flux de marchandises ou de capitaux, et des formes prises par les réseaux de communications qui individualisent les régions 


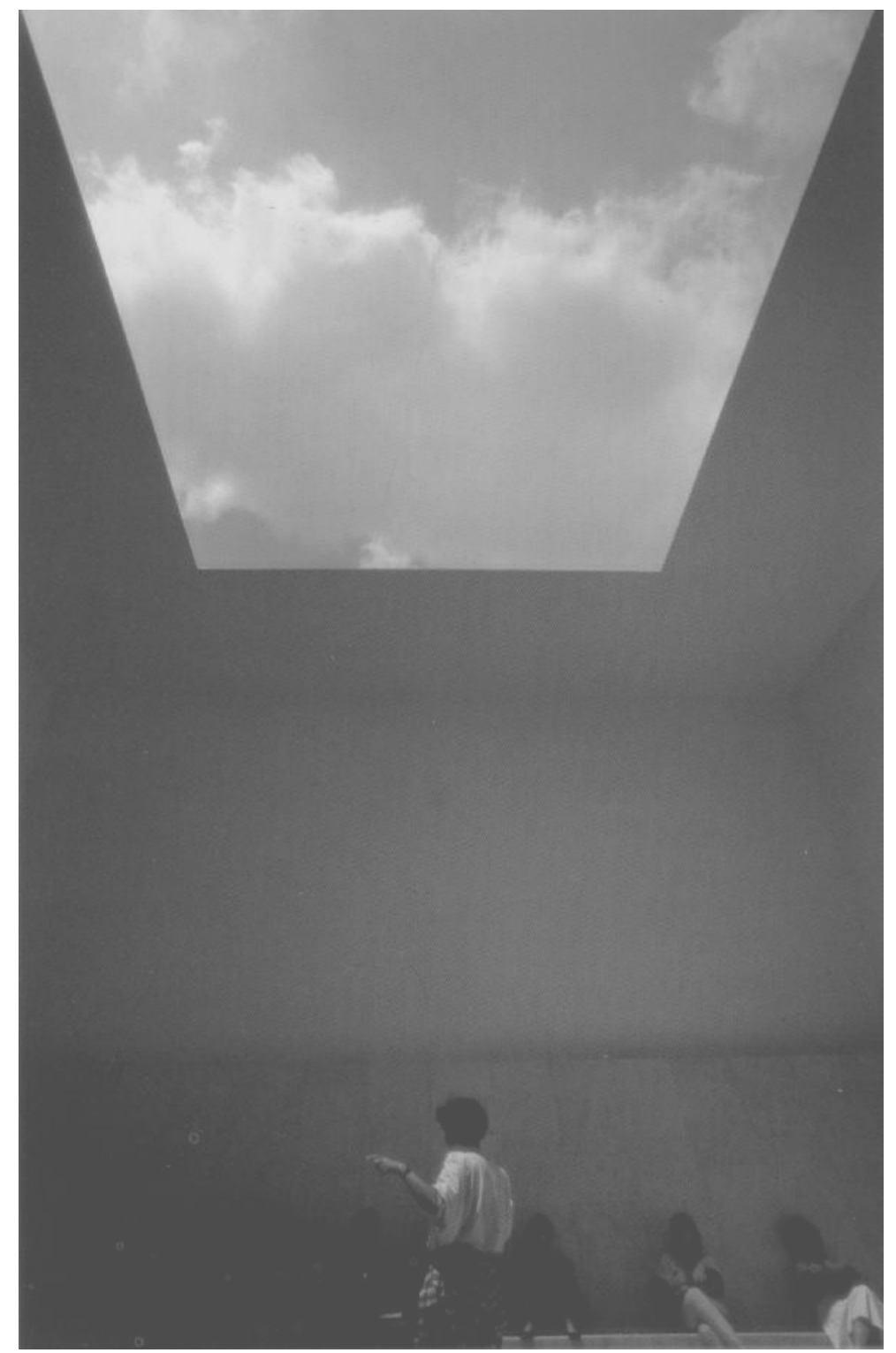

Fig. 4 - Un écran pour voir le monde: skyspace (installation), James Turrel, 1992, The Israel Museum.

Fig. 4 -Uma janela para o mundo, skyspace (instalação), James Turrel, 1992, The Israel Museum 


\section{EN GUISE DE COMMENTAIRES}

\section{Le peintre est un acteur géographique}

Le peintre est un acteur géographique, qui crée des relations sur l'espace d'une toile, et invente ainsi un modèle réduit d'une géographie possible. Son milieu est bien circonscrit, une toile blanche et plane. Grâce à ses outils et ses matériaux, il va la transformer à mesure qu'il y inscrit des signes visuels: la première ligne tracée découpe deux territoires; la première touche crée une tension; elles vont déterminer la suite de l'œuvre. De la même façon que la décision politique d'installer une capitale ou de monter une usine crée une dynamique de développement. Sur la toile, les signes visuels respectent, par les équilibres entre les masses et les couleurs, les règles plastiques de l'organisation d'un espace; de plus, ils obéissent à des conventions élaborées au cours des siècles et forment des images, figuratives ou non, qui sont autant de symboles caractéristiques d'une époque ou de l'idée que le peintre en transmet, volontairement ou non (FranCASTEL,1977; PanOvSKY,1981).

L'œuvre s'élabore progressivement, par essais et erreurs, chaque étape orientant la suivante. C'est une confrontation entre un individu libre, sensible, qui désire marquer le monde et un matériau, peinture et espace, qui lui résiste et lui impose un respect de ses lois. Le paysage que j'ai sous les yeux est également création, collective cette fois, incarnée dans la Nature, qui à la fois respecte et défie ses lois. La confrontation est risquée. Que l'idée l'emporte, il tombe dans l'anecdote, la propagande, la publicité. Que la main et le matériau dominent, c'est le décoratif, le gratuit ou la brute balbutiante qui apparaissent. Nul n'échappe au style d'une époque et aux modes du temps qui accentuent les déséquilibres qui flattent, et l'œuvre originale qui retrouve l'éternel et l'immuable prend du temps pour s'imposer. Le peintre sait qu'il vient de réussir quand il éprouve un «sentiment de jubilation enfantine devant l'inattendu». L'instant de grâce à travers lequel «le monde prend conscience de sa réalité visible» (DE BIASI, 1995) étonne le peintre lui-même et, comme toute découverte devient lentement une évidence. Un coin de pays devenu paysage réunit (BUREAU, 2001) à la fois une portion d'espace mystérieusement réussie et un regard qui la contemple et s'y reconnaît; et cet auteur ajoute que «des cours d'éveil de la sensibilité (...) cela vaudrait bien les sermons (...) des défenseurs de l'environnement».

\section{La peinture d'un paysage est ambiguë}

Le spectateur va apprendre à lire le tableau comme le géographe donne à voir ce qui se cache derrière les signes de l'organisation spatiale du monde. Il y a une double parenté entre la peinture et la géographie: d'une part, l'ensemble des rêves et des désirs d'une époque et leur traduction spatiale se reflètent 
matériellement et symboliquement sur les deux terrains; d'autre par,t il y a des analogies entre l'émergence et le développement de formes ou d'états particuliers dans des milieux différents; ces analogies ont des racines profondes dans les lois naturelles dont l'écho se prolonge dans l'esthétique elle-même: pensons par exemple aux ressemblances formelles entre un réseau de neurones, la peinture de Pollock et des réseaux de lieux géographiques.

Quand la peinture représente de manière réaliste l'organisation extérieure du monde, les deux espaces entrent en connivence. Il devient difficile de savoir si la disposition des objets sur une toile renvoie au langage plastique ou aux objets eux-mêmes. Cette ambiguïté de la peinture est souvent suggérée sur les tableaux par la présence d'un spectateur, d'une fenêtre ouverte sur l'extérieur, d'un miroir ou même d'un tableau du tableau. Que signifie au juste ce jeu entre un pays vu et sa représentation sur le tableau? Lequel sert de modèle? (CAUQuelin, 2000).

Si l'image se confondait avec ce dont elle est l'image, avec la Nature, alors le paysage peint ne serait qu'un beau simulacre parfaitement superflu; nous pourrions légitimement parler de paysage «naturel».Cette vue naïve est facilement dépassée quand on réalise l'énorme travail nécessaire pour faire exister un paysage. "Ceci n'est pas une pipe» nous rappelle Magritte! C'est que les peintres, avec la perspective, ont fabriqué une machine qui ordonne et incarne le monde, qui montre combien la Nature est bien faite. Leur vision subsiste et s'est élargie avec l'utilisation de nouveaux procédés pour inventer de nouveaux paysages tout aussi peu «naturels» que les anciens. Le naturaliste choisit les sites de l'écosystème avant de le photographier et dépasse ainsi la simple théorie des rapports entre les éléments du système à démontrer, dès lors qu'il s'incarne dans un ordre spatial idéal qu'on ne rencontre que là. Les premières photographies scientifiques des pics montagneux ont probablement mieux saisi leur «sublime» (Roger, 1997), leur majesté terrifiante que les peintres, tout en élargissant leurs essais. Et la photo ordinaire du pauvre touriste ressemble rarement à celle de la belle revue en couleur, qui a demandé des mois de recherche d'éclairage et de cadrage du site pour transformer ce dernier en paysage attrayant. Et mettez-vous à la place du géographe qui prépare une excursion où il va montrer des "paysages régionaux»: quel travail énorme pour dénicher le point de vue qui découvre harmonieusement l'intuition qu'il a fini par acquérir du pays à force de l'étudier.

À l'inverse, beaucoup de paysages géographiques se sont eux-mêmes transformés pour se conformer à l'idée d'un tableau. Jadis les jardins ou les parcs mais, maintenant encore, le paysagement des lieux écologiques ne peut pas se passer d'une simulation qui montre et qui ordonne et, dans la campagne, on cache les pylônes électriques, on rase des constructions et on déplace des forêts, en partie pour paraître comme un beau tableau, par fierté mais aussi pour se vendre comme affiche touristique (DAGOGNET, 1982; MARCLEL, 1989). Les éléments du pays vu et représenté sont choisis pour illustrer une histoire exemplaire ou sa conception du monde tout en respectant un équilibre esthétique. 


\section{CONCLUSIONS : L'ARTISTE EST UN MÉDIATEUR ENTRE NOUS ET LE MONDE}

L'artiste entre en phase avec son environnement en le reproduisant; il agit comme médiateur entre nous et les objets et nous donne à voir des relations symboliques et affectives. Habiter le monde ne se limite pas à exploiter ses ressources; la cartographie picturale, qui a d'ailleurs bien des points communs avec la cartographie scientifique, vient la compléter et aider à comprendre l'espace mental d'une époque, qui en a façonné la géographie (PIVETEAU,1989). Il ne faudrait cependant pas oublier que cet espace imaginaire est biaisé: il est ici question de la pensée du pouvoir ou de l'élite à qui plaît le tableau, religion, noblesse, capital ou expert, et pas de celle de l'armée de l'ombre qui a façonné la terre. Le nombre de lieux choisis par les peintres est restreint: on connaît l'attrait des fontaines de Tivoli, de la forêt de Fontainebleau, du port de l'Estaque pour les fauves, des vues de Charlevoix ou des cascades autour de Québec... Voilà un beau sujet d'étude pour un géographe que celui des lieux élus et de leur signification pour la discipline! Le très beau livre de Schama (Schama, 1999) nous introduit dans l'univers des mythes collectifs et des pay-sages idéaux, des hauts lieux historiques, poétiques et symboliques, pour lesquels on s'est battu et qui ont aussi fondé la géographie.

L'artiste utilise le monde pour s'exprimer et l'étude de quelques ouvres ne donnerait qu'un portrait très incomplet d'une époque. La littérature, les dessins, les cartes aident à en extraire l'esprit; le travail semble plus facile avec l'existence du musée «imaginaire» qui réunit par la photographie toutes les œuvres du passé. Ajoutons qu'aucun siècle n'est caractérisé par une pensée unique et qu'il y a toujours une juxtaposition entre l'utilisation de procédés anciens et une création plus moderne; beaucoup d'essais avortent et les œuvres les plus caractéristiques sont souvent jugées à partir de leur postérité, donc désignées à posteriori.

Si la fréquentation des arts est ainsi «utile» pour le scientifique, ce serait cependant une faute que de rabaisser les tableaux ou les livres au seul état de documents visuel, historique et social, sans en profiter pour jouir de leur présence physique et en faire une fête de l'œil et de l'esprit. C'est le danger des collections supervisées par les spécialistes des sciences humaines qui créent un fourre-tout d'où toute notion de valeur est écartée au profit d'un témoignage souvent dérisoire. Réduire des œuvres à des documents historiques, sociologiques, géographiques... aboutit actuellement à présenter dans les musées des simples documents comme des œuvres, à occulter la nature profonde de ces dernières. Ce "gouffre inquiétant de la perception esthétique» (SINAÏ, 1997) n'est pas sans rappeler l'absence d'artistes dans les nombreux comités qui se penchent sur le paysagement fragmentaire actuel; et une géographie privée de ses références culturelles deviendrait rapidement incompréhensible.

L'artiste partage sa perception avec le spectateur, il la communique; il essaie à la fois de donner à voir le monde et de dominer, serait-ce avec des images ou 


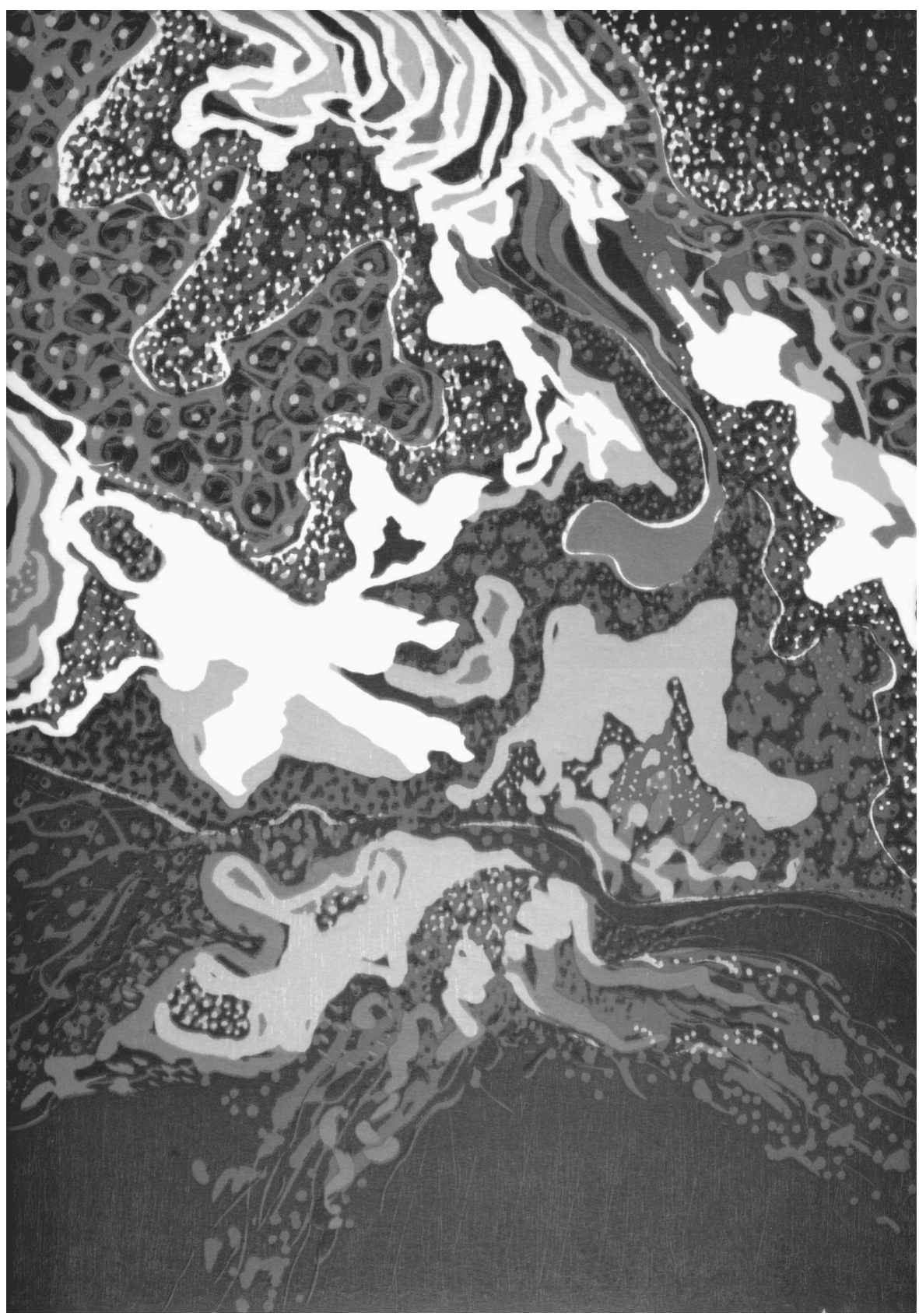

Fig. 5 - Turbations picturales, extrait d'une gravure «Oka III», René Derouin, $1981,32 \times 33 \mathrm{~cm}$, collection privée.

Fig. 5 - Turbações pictóricas, extracto de uma gravura "Oka III", René Derouin, 1981, $32 \times 33 \mathrm{~cm}$, colecção particular. 
des mots, la peur fondamentale d'être; c'est une des rares réponses possibles à l'absurdité et à la mort. L'autre réponse est celle de la science qui explore collectivement le «comment», le fonctionnement de la Nature, pour la maîtriser et être plus libre, mais sans trop savoir pourquoi. Les deux approches sont complémentaires en géographie, ne serait-ce que pour unir la part des déterminismes ou des enchaînements quasi inévitables et la part des rêves et des symboles dans la compréhension de l'organisation des territoires.

\section{BIBLIOGRAPHIE}

Barre, A. ; Flocon, A. (1967) - La perspective curviligne, Flammarion, 220 p.

Berque, A. (1995) - Les raisons du paysage, de la Chine antique aux environnements de synthèse, Hazan,192 p.

Bureau, L. (2001) - L'idiosphère, L 'Hexagone, 238 p.

CARLI, E. (1980) - Le paysage dans l'art, F. Nathan (trad.), 320 p.

Catalogue de l'exposition Nicolas Poussin (1994) - Galeries Nationales du Grand Palais, Paris.

Cauquelin, A. (2000) - L'invention du paysage, P.U.F.,181p.

CLARK, K. (1988) - L'art du paysage, Gérard Monfort, 189 p. (trad.).

Dagognét, F. ed. sc.(1982) - Mort du paysage? Actes du Colloque de Lyon, Éd. Champ Vallon (13 articles).

De BIASI, P.-M.(1995) - Le tableau, terre inconnue, Diogène, 169, janvier-mars: 89-99.

Deroin, R.(1997) - L'espace et la densité, L'Hexagone Ed., 236 p.

Francastel, P. (1977) - Peinture et Société, Denoël-Gonthier, 362 p.

Friedman, M., éd. sc. (1994) - Visions of America, In H.N. Abrams Inc., The Denver Art museums and the Columbus Museum of Art (collectif d'articles, $256 \mathrm{p}$ )

Friedrich.C.; CARUS C. (1988) - De la peinture de paysage, Klincksieck, Paris [traduction de l'original allemand, circa 1810].

GRIMALDI, N. (1983 ) - L'art ou la feinte passion, P.U.F., 300 p.

Lнот,P. (2000) - Peinture de paysage et esthétique de la démesure, L'Harmattan, 254 p.

Lhote, A.. (1948) - Traité du paysage, Floury, 221 p.

Marcel, J. éd. sc. (1989) - Composer le paysage, Éd. Champ Vallon (21 articles).

Mcshine, K., éd. sc. (1994) - The natural paradise; painting in America 1800-1950, New York Graphic Society, The museum of Modern Art, 180 p.

Panovsky, E. (1981) - La perspective comme forme symbolique, Les Éditions de Minuit, 273 p. (trad.)

Piveteau, J. (1989) - Les tableaux des peintres pour notre compréhension de l'espace. In «Yves ANDRÉ et al., Représenter l'espace, Anthropos, Paris.

Randolph, S. (1994) - Ambrogio Lorenzetti : the Palazzo publico, Siena, G. Braziller, N.Y., 103 p. 
Roger, A. (1997) - Du «pays affreux» aux sublimes horreurs. In Le paysage et la question du sublime, Réunion des musées nationaux et Musée de Valence :187-197.

SAuvagéot, J. ed. sc. (1993) - Les échelles du paysage, Presses Universitaires de Rennes, 100 p. (collectif d'articles).

SIMOËNS, J.-C. (1994) - Le voyage en Italie, J.C. Lattes, 275 p.

Schama, S. (1999) - Le Paysage et la Mémoire, Seuil, 722 p.

SINAÏ, A. (1997) - L'art contemporain face à l'histoire, Esprit, mars-avril: 71-82,

TRÉPANIER, E. (1997) - Le paysage au Québec, 1910-1930: un genre aux multiples enjeux. In Le paysage au Québec, Musée du Québec:11-34. 